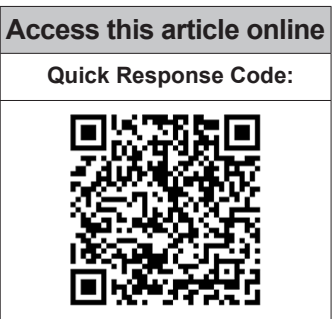

Website:

www.jponline.org

DOI:

10.4103/JLP.JLP_19_19
Department of Pathology and Laboratory Medicine, AlIMS, Bhubaneswar

Odisha, India

Address for correspondence: Dr. Gaurav Chhabra, Department of Pathology and Laboratory Medicine,

AlIMS, Bhubaneswar, Odisha, India

E-mail: gauravchhabra 2001@gmail.com

Submission: 31-01-2019 Accepted: 22-03-2019

\title{
Exflagellation of Plasmodium vivax in peripheral blood: An uncommon finding and its significance
}

\author{
Mukund N. Sable, Gaurav Chhabra, Shruti Mishra
}

\section{Abstract:}

Malaria continues to be a major public health problem. The life cycle of malaria is completed in two hosts Anopheles mosquito - definitive host and humans - the intermediate host. Exflagellation of microgametocyes in the life cycle of Plasmodium vivax occurs in mosquitoes and is rarely seen in human peripheral blood. Less than 15 occurrences of exflagellated microgametocyte of Plasmodium species have been reported to date. The appearance of exflagellated microgametes in human blood may pose a diagnostic dilemma due to its resemblance with other hemoparasites such as Borrelia and Trypanosoma.

Key words:

Exflagellation, hemoparasites, malaria, microgametocyte, Plasmodium

\section{Introduction}

$\mathrm{M}$ alaria is a major public health issue, particularly in Africa and Southeast Asia. It is caused due to infection with Plasmodium protozoa. The life cycle of Plasmodium species is complex and multistage, with sexual stage occurring in infected female Anopheles mosquito (definitive host) and asexual stage occurring in humans (intermediate host). All the asexual stages of Plasmodium vivax such as ring forms, late trophozoites, schizonts, and gametocytes are observed commonly in human blood whereas, sexual stages such as exflagellation of microgametocyte and microgametes are rarely seen in humans. Less than 15 occurrences of exflagellated microgametocyte of Plasmodium spp. have been reported to date. Herein, we report a case of $P$. vivax infection with different stages of exflagellation and numerous exflagellated microgametes in addition to the ring forms of $P$. vivax in peripheral blood of a patient

This is an open access journal, and articles are distributed under the terms of the Creative Commons Attribution-NonCommercial-ShareAlike 4.0 License, which allows others to remix, tweak, and build upon the work non-commercially, as long as appropriate credit is given and the new creations are licensed under the identical terms.

For reprints contact: reprints@medknow.com with high-grade fever and its significance in the clinical laboratory diagnosis.

\section{Case Report}

A 70-year-old female patient presented in the emergency department with high-grade fever for the last 2 days. The patient's blood sample was sent to the laboratory in ethylenediaminetetraacetic acid (EDTA) vacutainer for complete hemogram analysis. On receipt, in the laboratory, the sample was run on XT4000i (Sysmex, Kobe, Japan) automated hematology analyzer. The hemogram analysis revealed total leukocyte count of $11.43 / \mu \mathrm{l}$ with a system generated flag of abnormal white blood cell scattergram, and no differential count was reported by the analyzer. The scatter plot revealed no clear-cut separation of neutrophil and eosinophil population in WDF channel on SFL (Side Fluorescence) versus SSC (Side Scatter) plot, indicating the presence of hemoparasite [Figure 1]. Since no differential was given with an abnormal flag, a peripheral smear (PS) review was necessitated. The PS was made and stained

How to cite this article: Sable MN, Chhabra G, Mishra S. Exflagellation of Plasmodium vivax in peripheral blood: An uncommon finding and its significance. J Lab Physicians 2019;11:161-3. 
with Leishman stain and examined under the microscope which revealed numerous ring forms, trophozoites and schizonts of $P$. vivax [Figure 2a]. In addition, multiple clusters as well as singly scattered thin, long, filamentous flagellae-like structures were observed outside the red blood cells which were approximately around 10-15 $\mu$ in length and having oval-shaped nucleus. The careful morphological examination made us suspect the presence of exflagellated microgametes of $P$. vivax. On further examination, different stages of exflagellation of microgametocyes with the initial

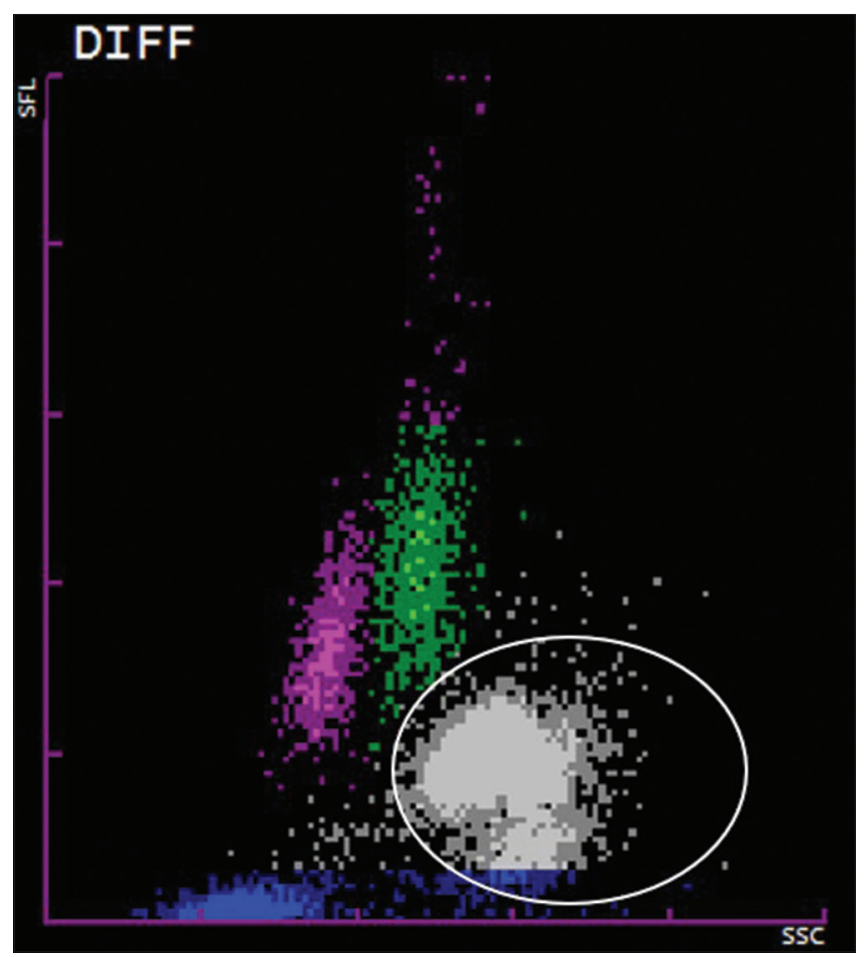

Figure 1: White blood cell scatter plot showing abnormal white blood cell scattergram (encircled population) stage of 6-8 flagellated microgametes arising out of microgametocyte [Figure $2 \mathrm{~b}$-e] followed by detached single exflagellated microgametes [Figure 2f] were found. To corroborate our finding, we made a fresh smear from finger-prick blood in which the exflagellated forms were absent.

\section{Discussion}

Exflagellation of Plasmodium microgametocytes occurs in mosquito and its appearance in human peripheral blood is an extremely rare phenomenon. In 1897, MacCallum ${ }^{[1]}$ first observed and reported this event in a patient with Plasmodium falciparum infection. On extensive search of English literature, Less than 15 occurrences ${ }^{[2-8]}$ have been reported in the form of case reports. To the best of our knowledge, this is the first case reported from India in which various stages of exflagellation from microgametocytes with resultant formation of microgamete have been found in human blood.

Microgametogenesis and exflagellation in vitro is dependent on many factors such as rise in $\mathrm{pH}, \mathrm{pCO}_{2}$, bicarbonate levels, and fall in temperature below that of the vertebrate host and anticoagulant. ${ }^{[9-11]}$ Mosquito exflagellation factor (MEF), a heat-stable molecule derived from mosquito's head and gut is considered as a most potent factor for in vitro exflagellation. ${ }^{[12]}$ The activity of MEF is dependent on the $\mathrm{pH}$ and bicarbonate level. The $\mathrm{pH}$ of human blood is around 7.35-7.45, which is lower than $\mathrm{pH}$ of mosquito gut, which inhibits the microgametogenesis and exflagellation. It is hypothesized that a change in $\mathrm{pH}$ in the laboratory may be triggered, when the blood comes in contact with the atmospheric environment resulting a fall in $\mathrm{CO}_{2}$, ultimately raising the $\mathrm{pH}$. This scenario mimics the change, the gametocytes are exposed to when they reach

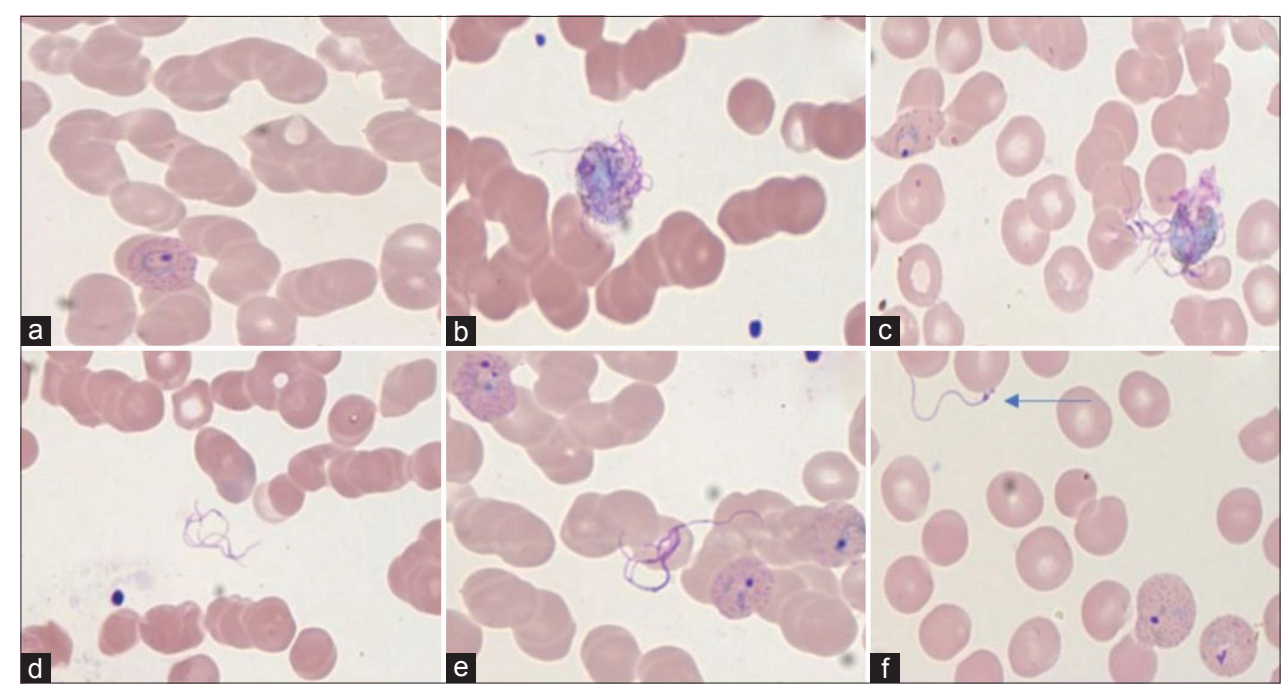

Figure 2: (a) Trophozoite of Plasmodium vivax (×1000; Leishman). ( $b$ and c) Exflagellation of microgametes from microgametocytes ( $\times 1000$; Leishman). (d-f) Exflagellated microgametes (×1000; Leishman) 
the gut of the mosquito following an infective meal, thus favoring exflagellation. ${ }^{[7]}$ In our case, exflagellation was not observed in smears prepared immediately through finger prick, thus confirming the fact that exflagellation was induced when the blood got exposed to atmospheric air for a longer duration, resulting in $\mathrm{pH}$ change.

Other reports suggest that phosphodiesterase inhibitors and the use of caffeine can induce this phenomenon in vitro; however, the mechanism is not clear. ${ }^{[12,13]}$ Solarte et al. suggested that the use of certain anticoagulant-like heparin induces exflagellation by causing a $\mathrm{pH}$ change, while EDTA inhibits it by preventing $\mathrm{pH}$ change, consumption of $\mathrm{Ca}^{2+}, \mathrm{Mn}^{2+}$, and $\mathrm{Mg}^{2+}$, which further prevents the activation of enzymes required for exflagellation. ${ }^{[14]}$ EDTA-induced prevention of flagellation is questionable, as majority of cases published reported exflagellation in the blood preserved with EDTA as seen in our case.

Due to its rarity, the presence of exflagellated microgametes in peripheral blood may pose a diagnostic dilemma, particularly for an inexperienced observer because of its resemblance with other hemoparasites such as Borrelia, Microfilaria, or Trypanosoma or it may be overlooked as a staining artifact. However, with careful morphological examination, these can be differentiated as the exflagellated form of Plasmodium occur as thin filamentous structure measuring approximately $10-15 \mu$ in length with an oval-shaped dark blue nucleus. In contrast, Borrelia is 5-20 $\mu$ in length with a spiral shape and lack nuclei and Trypanosoma has an undulating membrane, kinetoplast, and a nucleus. ${ }^{[3,4]}$

\section{Conclusion}

The presence of exflagellated microgametes in human peripheral blood is a rare phenomenon and this is possibly the third reported case from India, an endemic zone for malaria. Lack of knowledge of this poses a diagnostic challenge because of its resemblance with other hemoparasites. Careful morphological examination can help in correctly diagnosing and differentiating these forms from other hemoparasites.

\section{Declaration of patient consent}

The authors certify that they have obtained all appropriate patient consent forms. In the form the patient(s) has/ have given his/her/their consent for his/her/their images and other clinical information to be reported in the journal. The patients understand that their names and initials will not be published and due efforts will be made to conceal their identity, but anonymity cannot be guaranteed.

\section{Financial support and sponsorship}

Nil.

\section{Conflicts of interest}

There are no conflicts of interest.

\section{References}

1. MacCallum WG. On the flagellated form of malaria parasite. Lancet 1897;2:1240.

2. Gerber JE, Ukena TE, Cote L, Wyllie JM, Winn WC Jr. Exflagellation of malarial parasites in human peripheral blood. J Clin Microbiol 1981;13:236-7.

3. Tembhare P, Shirke S, Subramanian PG, Sehgal K, Gujral S. Exflagellated microgametes of Plasmodium vivax in human peripheral blood: A case report and review of the literature. Indian J Pathol Microbiol 2009;52:252-4.

4. Prasad CS, Aparna N, Harendra Kumar ML. Exflagellated microgametes of Plasmodium vivax in human peripheral blood: An uncommon feature of malaria. Indian J Hematol Blood Transfus 2011;27:104-6.

5. Balestra B, Carnino L. Images in clinical medicine. In vitro exflagellation of Plasmodium vivax. N Engl J Med 2016;375:e27.

6. Diallo MA, Diongue K, Ndiaye D. Plasmodium falciparum exflagellation in a patient with ovalocytosis. Am J Med Sci 2017;354:68.

7. Arneth B, Keller C, Schaefer S. Malaria exflagellation in a human peripheral blood smear. IDCases 2017;10:51-2.

8. Weinstein MP, Alcid DV. Occurrence of exflagellation and microgametes in peripheral blood of a patient with malaria. J Infect Dis 1982;146:448.

9. Enger A, Strand ØA, Ranheim T, Hellum KB. Exflagellation of microgametocytes in Plasmodium vivax malaria: A diagnostic conundrum. Med Princ Pract 2004;13:298-300.

10. Carter R, Nijhout MM. Control of gamete formation (exflagellation) in malaria parasites. Science 1977;195:407-9.

11. Billker O, Shaw MK, Margos G, Sinden RE. The roles of temperature, $\mathrm{pH}$ and mosquito factors as triggers of male and female gametogenesis of Plasmodium berghei in vitro. Parasitology 1997;115 (Pt 1):1-7.

12. Nijhout MM. Plasmodium gallinaceum: Exflagellation stimulated by a mosquito factor. Exp Parasitol 1979;48:75-80.

13. Martin SK, Miller LH, Nijhout MM, Carter R. Plasmodium gallinaceum: Induction of male gametocyte exflagellation by phosphodiesterase inhibitors. Exp Parasitol 1978;44:239-42.

14. Solarte Y, Manzano Mdel R, Rocha L, Castillo Z, James MA, Herrera $S$, et al. Effects of anticoagulants on Plasmodium vivax oocyst development in Anopheles albimanus mosquitoes. Am J Trop Med Hyg 2007;77:242-5. 